Litinfinite Journal

ISSN: 2582-0400 [Online]

CODEN: LITIBR

Vol-1, Issue-1 ( $2^{\text {nd }}$ July, 2019)

Page No: $78-88$

DOI: 10.47365/litinfinite.1.1.2019.78-88

Section: Interview

\title{
An Interview with Poet Prabal Kumar Basu
}

\author{
Sreetanwi Chakraborty \\ Assistant Professor \\ Amity Institute of English Studies and Research \\ Amity University Kolkata \\ West Bengal, India. \\ Mail Id: schakraborty3@kol.amity.edu
}

\begin{abstract}
This is an interview with poet Prabal Kumr Basu who is a modern poet, having eighteen poetry books to his credit. He has carved out a definite name in the field of modern Bengali poetry and also has a book of verse drama to his credit. He has received notable accolades and prizes as part of his writing experience, and in this interview, he talks about his childhood, the most important and influential poets whose poetic imprints have inspired him. Moreover, he talks about world poetry as a part of this interview and also carries a very detailed analysis of the present condition of translation studies in the world. The interviewer also asks him as a part of his world tours for official work and also for poetry sessions. He recollects all the moments of poetic enrichment that have helped him in becoming a creatively affluent individual, and a worshipper of poetry.
\end{abstract}

Keyword: Interview, Poetry, Translation, Bookfair, Little Magazine

Poet Prabal kumar Basu was born on $21^{\text {st }}$ September, 1960, in Kolkata, the erstwhile Calcutta. His first poem was published in Samayanug Patrika. His first poetry reading session was in Baker Hall in Presidency College, Calcutta. It was one of the biggest poetry festivals organized by Prabaha in Calcutta then. His first poetry book was Tumii Pratham. He has edited a book Signposts Bengali Poetry since Independence. In 2003 he formed Calcutta International Foundation for Art, Literature and Culture which consisted of famous artists, theatre personalities, poets and other culturally-enriched individuals from all walks of life. In 2004 , on the eve of $50^{\text {th }}$ year celebration of Sahitya Akademi, Basu was invited to Bombay for All India Poetry Festival, where he participated in reading sessions. He co-edited a volume of Krittibas that was especially dedicated to write-ups by notable artists. In 2005, Basu received much fame and accolades, along with the West Bengal Bangla Akademy Puraskar, for his poetry book Jamon kore gaichhe Akash. In this same year he was invited to the Third International Poetry Festival in Wellington, New Zealand. Mail Id: prabalkumar@gmail.com

Note: This is an interview conducted in Bengali and there is no citation or works cited required. It is a live interview taken by Sreetanwi Chakraborty, Chief-Editor of Litinfinite Journal. 
CODEN: LITIBR

Vol-1, Issue-1 (2 $\left.{ }^{\text {nd }} J u l y, 2019\right)$

Page No: $78-88$

DOI: 10.47365/litinfinite.1.1.2019.78-88

Section: Interview

\section{বিশিষ্ট কবি প্রবাল কুমার বসুর সাক্ষাৎকার নিয়েছেন অধ্যাপক শ্রীতন্বী চক্রবর্তী।}

শ্রীতন্বী চঞ্রবর্তীঃ প্রথমেই অনেক অভিনন্দন জানাই, অন্তরালের গল্পকথা প্রকাশিত হওয়ার জন্যে। বইটি সম্বন্ধে আমাদের পাঠকদের কিছু বলুন।

প্রবাল কুমার বসুঃ আসলে আমি যে পরিবেশে বড় হয়ে উঠেছি, যেভাবে সংস্কৃতি জগতটাকে দেথেছি, এবং দেথার বেশিও অনেকটা যা দেখিনি, সে সম্পর্কে শুনেছি, সেই কথাগুলোর সূত্র ধরে আমার মনে হয়েছিলো এগুলোকে কোথাও একটা একত্রিত করা দরকার। তা নাহলে এগুলো হারিয়ে যাবে। এবং আমাদের এই চলমান সংস্কৃতিতে, বরং বলা ভালো চলমান সংস্কৃতির বিবর্তনের পথে এই ঘটনাগুলোর এক উল্লেথযোগ্য অবদান রয়েছে। সেই পরিস্থিতিতে এই লেথাগুলো আমি অনেক দিন ধরেই লিথছিলাম, বিভিন্ন জায়গায় বেরোষ্ছিলো, কোথাও কোথাও বক্তৃতাও দিচ্ছিলাম, সেগুলো একত্রিত করা হলো, "its more a documentation than anything else, which is likely to help future researchers I"

শ্রীতন্বী চঞ্র্তীঃ আপনার কাব্যিক অনুপ্রেরণার বিষয়ে আমাদের কিছু বলুন।সাহিত্য জগতের নক্ষত্ররা, যেমন দেবকুমার বসু, শক্তি চট্টোপাধ্যায় এবং সুনীল গঙ্গোপাধ্যায় আপনাকে কি কোনোভাবে প্রভাবিত অথবা অনুপ্রাণিত করেছিলেন?

প্রবাল কুমার বসুঃ Poetic inspiration comes from within, it has nothing to deal with anyone else I But, the very fact that I started writing poetry was due to an incident, (আমার তো বাংলায় বলার কথা) আমাদের এক পারিবারিক বন্ধু ছিল। আমার বাবার বন্ধু। উনি ডাক্তার ছিলেন, কিন্ত কোনোদিন ডাক্তারি করেননি। উনি মোমবাতির ব্যবসা করতেন, এবং সেই সময় কলকাতায় আকছার লোডশেডিং হওয়ার কারণে তিনি মোমবাতির ব্যবসা করে অনেক টাকা রোজগার করেছিলেন। বিদেশীদের মতো চেহারা ছিলো ওনার। নিরামিযাশী এবং থুব sophisticated ভদ্রলোক ছিলেন। প্রত্যেকদিন উনি দুকিলো মাংস কিনতেন এবং বিভিন্ন রকমের পদ রান্না করতেন। তার মধ্যে থাকতো বাঁধাকগির মাংস, ফুলকপি দিয়ে মাংস, দুধ দিয়ে মাংস, আসলে তিনি নানারকম experiment করতে ভালোবাসতেন। ওনার একজন রান্নার ঠাকুর ছিলো। উনি তাকে রোজ নির্দেশ দিতেন কোন রান্নায় কী মশলা এবং কী উপাদান 
Litinfinite Journal

ISSN: 2582-0400 [Online]

CODEN: LITIBR

Vol-1, Issue-1 (2 $\left.2^{\text {nd }} J u l y, 2019\right)$

Page No: $78-88$

DOI: 10.47365/litinfinite.1.1.2019.78-88

Section: Interview

কতটা পরিমান দিতে হবে। ওনার বাড়িতে অতিথি এলে উনি সব রকম মাংসের পদ ছোট ছোট বাটিতে পরিবেশন করতেন।

আমাকে থুব ভালোবাসতেন ভদ্রলোক। এই ভদ্রলোক যথন মৃত্যু পথযাত্রী, কলকাতায় মেডিকেল কলেডে ভর্তি, তথন আমাকে দেথতে চাইলেন। আমি সেইসময় ক্লাস ইলেভেনে পড়ি, দেথলাম উনি মুমূর্মু অবস্থায় রয়েছেন। আমাকে দেথে উনি বললেন "তুই এথন লিথছ্ছিস?" আমি বললাম "না"। বললেন “আমি একসময় লিথতাম”। এই বলে তেল চিটচিটে বালিশের তলা থেকে একটা লেথা বার করলেন, বললেন "দেথ, রবীন্দ্রনাথের সাথে আমার লেথা ছাপা হয়েছে"।

তার কয়েকদিন পরেই উনি মারা গেলেন। তাঁর সেই পরিতৃপ্রিটা আমাকে সেদিন ভীযণভাবে নাড়া দেয়। তারপর মনে হয় আমারও তো জীবনে একটা achievement হতে পারে যদি (োনোদিন এরকম কারো সঙ্গে একটা লেথা ছ্বাপাতে পারি। আমাদের সময় কবি শক্তি চট্টোপাধ্যায় ঈশ্বরের সমতুল্য। বাবাকে বললাম কবি শক্তি চট্টোপাধ্যায়ের সাথে আমার একটা লেথা ছাপাতে হবে। তথনো কোনো কবিতা লিথিনি ছাপাবার উদ্দেশ্যে। यাই লিথি বাবার কিছুতেই পছনন্দ হয় না। অবশেশে আমার একটা লেথা বাবার পছন্দ হলো, বললেন "এটা ছাপানো যেতে পারে"। ছাाা হলো, তথন বক্ষ দুরুদুরু। পত্রিকা থুলে দেথি আমার লেথা ছাপা, কিন্ত আমি তথন শক্তির লেথা থুঁজছি। কিন্তু শক্তির লেথা নেই, চরম হতাশা। এরকম তো কথা ছিলো না!

তথন বাবা বললেন তুই আর একটা কবিতা লেথ। সেটা যথন বেরোলো তথন 'প্রবাহ' পত্রিকা সারাদিন ধরে প্রেসিডেন্সি কলেজের বেকার হলে সারা বাংলা কবি সন্মেলন করতো। তথন এথনকার মতো এতো কবি সম্মেলন হতো না। বড় বড় কবিদের সমাহার, সেখানে আমার কবিতা পড়ার কথা ছিলো না। তথন কে যেন ডাকলেন, বললেন "এই ওকে ডাকো তো, ও তো সর্বকনিষ্ঠ কবি যার লেথা ছাপা হয়েছে"। পরে যথন কবিতা গড়ে বেরোচ্ছি, তথন কে একজন আসার গাল টিপে দিচ্ছে, তাকিয়ে দেথি কবি সুভাষ মুথোপাধ্যায়।ওনাকে আমি সুভায জ্যাঠা বলতাম। পরে আরো একজন এসে আমায় জড়িয়ে ধরলেন, দেথি কবি অমিতাভ দাশগুপ্ত। তথন মনে হলো ব্যাগারটা মন্দ নয়। যদি আট দশ লাইন কবিতা লিথে এতো মানুমের ভালোবাসা পাওয়া যায়, তাহলে কবিতাই লিথি। So that was the inspiration। মানুমের ভালোবাসা পাওয়া, যা পেয়ে চলেছি এখনো।

শীরীন্বী চঞ্রবর্তীঃ এটা কি সত্যি যে আभনি ছোটবেলা থেকে অনেক সুযোগ পেয়েছেন, যা পরে আभনাকে নিজেকে তুলে ধরতে সাহায্য করেছে?কোন স্মরণীয় অভিজ্ঞতা যা আপনি পাঠকদের জানাতে চান?

Note: This is an interview conducted in Bengali and there is no citation or works cited required. It is a live interview taken by Sreetanwi Chakraborty, Chief-Editor of Litinfinite Journal. 


\section{Litinfinite Journal}

ISSN: 2582-0400 [Online]

CODEN: LITIBR

Vol-1, Issue-1 ( $2^{\text {nd }}$ July, 2019)

Page No: $78-88$

DOI: 10.47365/litinfinite.1.1.2019.78-88

Section: Interview

প্রবাল কুমার বসুঃ I had a very enriched childhood/ I differentiate between being part of a chequered and an enriched childhood. কলেজে পড়ার সময়টাকে তো চাইল্ডহুড বলা যাবে না। স্কুলে গড়ার সময় আমি একদিন প্রেমেন্দ্র মিত্রের বাড়িতে গিয়ে তার নাতনির প্রেমে গড়ে গিয়েছিলাম। এবং সেও আমার প্রেমে পড়ে গিয়েছিলো। Enriched এই অর্থে বললাম যে, জীবনে আমি থুব বড় বড় মানুমের সান্ন্যিধ্য পেয়েছি।যেমন নাট্যকার মন্মথ রায়, সুনীতি চটোপাধ্যায়, প্রেমেন্দ্র মিত্র এবং আরো অনেকে। সেই অর্থে বলা যেতে পারে আমার মনন এবং মানসিকতা কে তারা অনেকাংশেই অনুপ্রেরণা যোগাতে সাহায্য করেছেন। আমার বয়েসি এথন অনেকেই যাঁরা বেঁচে আছেন, I feel privileged যে ওরা কেউই এদের সাহচর্য গাননি। মানে সমরেশ বসু onward হয়তো পেয়েছেন তবে তাঁদের আগের প্রজন্ম, অর্থাৎ, সুনীতি বাবু, সুকুমার সেন যাঁদের সাহচর্য আমি থুব ছোটবেলা থেকে পেয়েছি তাঁদের অনেকেই পায়নি।

শ্রীতন্বী চঞ্র্তীঃ ১৯৮৩ সালে সেপ্টেম্বর মাসে আभনার প্রথম বই ‘তুমিই প্রথম’ প্রকাশিত হয়। সেই মুহূর্তটি কেমন ছিলো?

প্রবাল কুমার বসুঃ এটা তো থুব উত্তেজনার একটা ব্যাগার। থুব স্বাভাবিকভাবেই প্রত্যেকেরই নিজের প্রথম বই নিয়ে একটা উত্তেজনা থাকে। আমার প্রথম বই নিয়ে একটাই ভাবনার বিযয় ছিলো- ততদিনে শক্তি চট্টোপাধ্যায়কে আমি আমার গুরু বলে মেনেছি। আমার একান্ত ইচ্ছে ছিলো শক্তির প্রথম বইয়ের প্রচ্ছদ যে শিল্পী করেছিলেন, প্রথম বই যে প্রকাশনা দগ্তর থেকে হয়েছিল আমার প্রথম বইয়ের প্রচ্ছদও সেই শিল্পী ও বই প্রকাশকই করবে। यদিও ততদিনে সেই প্রকাশনা দগ্তর উঠে গিয়েছে। কিন্ত সেই প্রকাশকের হাত দিয়েই আমার প্রথম বই বেরিয়েছিল এবং শক্তির প্রথম প্রকাশনার প্রচ্ছদ যে করেছিল সেইই আমার বইয়ের প্রচ্ছদ করেছিল।

শ্রীতন্বী চর্রবর্তীঃ আপনাকে এককথায় বিশ্ব-পর্যটক হিসেবে অভিহিত করা যেতে পারে। কর্ম সূত্রেই হোক অথবা কবি পরিচয়ে, আপনি নিত্যনতুন কাব্যিক ভাষার অনুসন্ধান দিতে থাকেন নিরন্তর। এই বিশ্ব-ভ্রমন আগনার কবিতাকে কিভাবে অনুপ্রেরণা যুগিয়েছে?

প্রবাল কুমার বসুঃ অবশ্যই, এইযে বিভিন্ন মানুষদের দেথা, তাদের সাথে আলোচনা করা, এবং আমি বেশিরভাগ সময়েই যথন যে দেশে যাই, চেষ্টা করি সেই দেশের কোনো কবির সঙ্গে, আমার পরিচয় সরাসরি থাকুক অথবা বিশ্বময় অনেক কবিবন্ধু রয়েছে যাঁদের মারফত যোগাযোগ করে আমি চেষ্টা করি কিজ্ভুটা সময় বের করে তাদের সঙ্গে কাটাতে। আমি ইরানে গিয়েও এইভাবে কবিসঙ্গ করেছি। জাগান, নিউজিল্যান্ড, অস্ট্রেলিয়া তে আমার অনেক কবি বন্ধু রয়েছে যাঁদের সঙ্গে সররাসরি আমার এথনো কাব্যিক আদানপ্রদান হয়ে থাকে। আমাকে মূলত বিভিন্ন দেশ মুরতে হয় আমার কর্মসূত্রে। কিন্ত আমি তার মধ্যেও সময় বের করে

Note: This is an interview conducted in Bengali and there is no citation or works cited required. It is a live interview taken by Sreetanwi Chakraborty, Chief-Editor of Litinfinite Journal. 
Litinfinite Journal

ISSN: 2582-0400 [Online]

CODEN: LITIBR

Vol-1, Issue-1 (2 $\left.{ }^{\text {nd }} J u l y, 2019\right)$

Page No: $78-88$

DOI: 10.47365/litinfinite.1.1.2019.78-88

Section: Interview

সেইসব দেশের কবিবন্ধুদের সাথে আলাপ আলোচনা করি যা পরবর্তীকালে আমাকে অনেক লেথার ক্ষেত্রেই

প্রভাবিত করে।

শীরীন্বী চঞবর্তীঃ কিছু সময়ে মনে হয় ‘এই যে আমি চলেছি’ এক গভীর নিঃসঙ্গতার প্রতীক, একটি বিচিএদৃকের মাধ্যমে তুলে ধরে শব্দ, ধ্ননি, বাক্য এবং চেতনা, যা আমাদের নিয়ে যায় এক অনস্তিত্বের অভিমুথে। এই শূন্যতার কারন কি?

প্রবাল কুমার বসুঃ Travelling to nothingness / We all travel to nothingness / We think that we are travelling to somewhere, to some destination, but that destination is actually nothingness। যে কারণে আমার একটি বই রয়েছে 'আপনাকেই ঠিক করতে হবে গন্তব্য'। So, এই travelling টা भুরোটাই তো journey। ট্রাভেলিং is part of the journey / That's all, nothing more, and it depends on how one takes ingredients from that journey, for his or her own enrichment / So I think I could take a lot.

শ্রীতন্বী চঞ্র্তীঃ আभনি কবিতার ক্ষেত্রে সুবন্দিত। পশ্চিমবঙ্গ বাংলা একাডেমি পুরস্কার এবং গৌরী ভট্টাচার্শ স্মৃতি পুরস্কার পাওয়ার অভিজ্ঞতা (ক্ন ছিলো?

প্রবাল কুমার বসুঃ গৌরী ভট্টাচার্শ স্মৃতি পুরস্কারটা ছিলো আমার প্রথম বই এর জন্য। সেইসময় এই পুরস্কার এর জন্যে আরো কয়েকজন প্রতিযোগী ছিলেন যাঁরা এখন বেশ স্বনামধন্য। যাইহোক পুরস্কার টা আমিই পেয়েছিলাম। একজন কবির প্রথম বই পুরস্কার পাওয়া এবং এই পুরস্কার এর সেই সময় একটি অর্থমূল্য ছিলো। 1984 সালে এই পুরস্কার এর অর্থমূল্য ছিলো পাঁচ হাজার টাকা। একটি বড় অনুন্ঠানের মাধ্যমে অন্নদাশঙ্কর রায়ের হাত দিয়ে এই পুরস্কারটা দেওয়া হয়েছিল। প্রথম গৌরী ভট্টাচার্য সৃৃতি পুরস্কারটা আমিই গাই। সুনীল গঙ্গোপাধ্যায় দ্বিতীয় বছ্র এই পুরস্কারটি গান। তাই আমি বরাবর সুনীলদাকে বলে এসেছ্ছি 'আমি এই একটা বিষয়ে কিন্ত আপনার থেকে সিনিয়র। আমার পরে আপনি এই পুরস্কারটি পেয়েছেন। এছাড়া পশ্চিমবঙ্গ বাংলা একাডেমি পুরস্কারটি পেয়েছ্ অনেক বছ্র আগে। যে কোনো পুরস্কারই একটা স্বীকৃতি যা যে কোনো কবিকেই উদ্বুদ্ধ করে এবং তার বহুমূল্যবান প্রচেষ্টাকে স্বীকৃতি দেয়।

শ্রীতন্বী চঞবর্তীঃ আপনার কি মনে হয় যে সারা বিশ্বব্যাপী আন্তর্জাতিক কবি সন্মেলন, little magazine উৎসব এবং বইমেলার দ্বারা নতুক পাঠক-পাঠিকাদের কাছে পৌঁছনো যায়? আপনি কি ভাবে এইসব সাহিত্য অনুষ্ঠানের মধ্যে দিয়ে আপানার পাঠকদের চাহিদা পুরন করেন?

প্রবাল কুমার বসুঃ দুটো আলাদা paradigm- international poetry festival এবং লিটল magazine উৎসব। আমি দীর্ঘদিন হোলো little magazine উৎসবে কবিতা পড়া ছেড়ে দিয়েছি। তার কারণ আমার মনে

Note: This is an interview conducted in Bengali and there is no citation or works cited required. It is a live interview taken by Sreetanwi Chakraborty, Chief-Editor of Litinfinite Journal. 


\section{Litinfinite Journal}

ISSN: 2582-0400 [Online]

CODEN: LITIBR

Vol-1, Issue-1 (2 $\left.{ }^{\text {nd }} J u l y, 2019\right)$

Page No: $78-88$

DOI: 10.47365/litinfinite.1.1.2019.78-88

Section: Interview

হয় এথানে কবিতার যথাযথ সম্মান দেওয়া হয়না বা সম্মান দিয়ে কবিতা শোনার কেউ থাকেনা। এটা একটা হুগুগে প্রক্রিয়া মাত্র। মাথা গুলিয়ে দেওয়ার মতো একটা ভিনিস। এই কারণে দশ বারো বছ্র, হয়তো তার বেশীও হতে গারে আমি এই ধরণের অনুষ্ঠানে কবিতা পড়া বন্ধ করে দিয়েছি। আন্তর্জাতিক কবিতা সম্মেলনগুলোর আলাদা মাত্রা আছে যেগুলো শুধুমাত্র কবিতার জন্যই অনুষ্ঠিত হয়। আমার বিচিত্র সব অভিজ্ঞতা হয়েছে কয়েকবার। নিউজিল্যাণ্ডে ওরা একবার একটি পানশালাতে কবিতা পাঠের আয়োজন করেছিল। তার বাইরে সাতদিন আগে থেকে টাঙিয়ে দেওয়া হয়েছিল যে ঐদিন কবিতা পাঠ হবে এবং গানশালায় সেইদিন সবাইকে দশ ডলার এক্সট্রা দিতে হবে কবিতা শোনার জন্য। এই নয় যে আমি কবিতা ভালোবাসিনা, মদ থেতে ভালোবাসি, এবং যে যার মতো কবিতা পড়ছে পড়ুক তাও নয়। আমি দেথেছ্ ওয়েলিংটন-এর পানশালা ভরে গিয়েছিলো এবং দর্শকরাও দীর্ঘ আলাপচারিতা করেছিল কবিদের সঙ্গে। এটা একটা অদুুত অভিজ্ঞতা। এছাড়াও এরা গ্রন্থাগার এ কবিতা পাঠের অনুষ্ঠান করেছিল, এবং সেদিন গ্রন্থাগার এ অভাবনীয় ভিড় হয়েছিল।আগে থেকেই বোর্ড টাঙিয়ে বিজ্ঞাপন দিয়েছিলো।

এছাড়া আর একটা ঘটনা বলি। আমি যথন নিউজিল্যাণ্ডে কবিতা পড়ছিলাম (নিউজিল্যাণ্ডে সেই অনুষ্ঠানে আমিই ছিলাম সর্বকনিষ্ঠ।) দুটি মেয়ে কবিতা পড়ার শেশে আমার সাথে এসে আলাগ করলো। তারা বললো প্রতিমাসে শুক্রবার সারারাত ধরে তারা কবিতা গাঠের আয়োজন করে, একটা ঘরোয়া জায়গায়, আমি यদি সম্মত হই সেথানে যেতে তাহলে ওরা আমাকে যোগ্য সম্মান ও গারিশ্রমিক দেবে। আমাকে যাঁরা নিয়ে গিয়েছিলো, সেই উদ্যোক্তাদের অনুমতির প্রয়োজন ছিলো আমার যাওয়ার জন্যে। ওখানকারই

উদ্যোক্তাদের মধ্যে একজন কবি, তার সাথে আমার বন্ধুত্ব হয়ে গিয়েছিল কয়েকদিনের মধ্যেই, তাকে আমি কথাটা বললাম। সে বলল এটা সামান্য ব্যাপার, চলো। যাব কি করে? ওরা বলল আমরা ট্যাক্সি ভাড়া দিয়ে দিছি, ট্যাক্সি করে চলে আসুন।

আমি ভয় পেলাম, আমি চিনিনা, জানিনা, এরকম ভাবে ট্যাক্সি করে যেতে পারবো না। তথনো আমার বিদেশ যাওয়াটা অতটা রগ্তু হয়ে ওঠেনি আর কি। গিয়ে দেথলাম একটা একতলা বাড়িতে একদিকে একটা বারকাউন্টার রয়েছে এবং আর একটা ঘরে কবিতা পাঠ চলছে। ঘরের মধ্যে একটা চৌপায়া, অথবা তক্তপোয গোছের পাতা রয়েছে, তার উপরে একটা মাই(্রোফোন, সেথানে এক একজন কবি বসে, আধ ঘন্টা ধরে কবিতা পড়বে। কবিতা পড়ার গরে, দশ মিনিট করে interaction হবে, সেশন শেষ। ওই যে সময়টা হছ্,ে, ততফ্ণণ লোকে ওই বার-কাউন্টার থেকে মদ কিনে এনে ওখানে বসে মদ্যপান করতে পারবে। কিন্ত কেউ কোন কথা বলবে না। আমি দেথলাম, সবাই মনোযোগ দিয়ে কবিতাপাঠ শুনছে। একজনের পড়া শেষ হলে, বিরতি দশ মিনিট, ওই দশ মিনিটের সময় সবাই বেরিয়ে আসছে। ২০০৫ সালেই নিউভিল্যাণ্ডে বাড়ির ভিতরে ধূমপান নিষিদ্ধ ছিল। সবাই বাইরে এসে কথা বলছে, ধূমগান করছে, আবার ঘন্টা পরছে, আর দশ মিনিট পর অন্য জন পড়বে, আবার সবাই গিয়ে মদ নিয়ে বসে পড়লো চুপচাথ। 


\section{Litinfinite Journal}

ISSN: 2582-0400 [Online]

CODEN: LITIBR

Vol-1, Issue-1 ( $2^{\text {nd }}$ July, 2019)

Page No: $78-88$

DOI: 10.47365/litinfinite.1.1.2019.78-88

Section: Interview

এইটা আমার একটা দারুন প্রাগ্তি। এবং প্রায় সত্তর আশি জন তরুন ছেলে-মেয়ে, সবাই মোটামুটি কলেজইউনিভার্সিটি তে গড়ে, এটা আমার এক অসাধারণ কবিতাপাঠের আসর মনে হয়েছ্ছিলো।

শ্রীতন্বী চর্রবর্তীঃ আभনি বহুদিন ধরে কাব্যনাটক নিয়ে পরীক্ষা-নিরীক্ষা করছেন এবং লেথালিথি করছেন। আপনি কি কোনভাবে ‘মনোবাঞৃ এক বিন্দু জল’ এর দেবাংশুর মধ্যে নিজের আদল থুঁজে পান?

প্রবাল কুমার বসুঃ এটা হছে, all wishful wishes। একটা particular form-এ নিজেকে প্রকাশ করতে করতে, যথন একজন ক্লান্ত হয়ে পরে, তথন একজনের মনে হয়, আরো একটা ফর্মে কিছু প্রকাশ করতে পারলে ভালো হতো। তথনই আরেকটা genre থুঁজতে যাওয়া। এই থুঁজতে গিয়েই আমি গল্প লিথেছ্ কথনো, কিন্ত কাব্যনাটকটায় মনে হয়েছে, যেন আমি যেটা বলতে চাইছ্ছি, যেটা আমি কবিতায় বলতে পারছি না, সেটা কাব্যনাটকে আমি অনেক ভালোভাবে বলতে পারছি।

And I as a character, I always prefer to travel the path less travelled। কাব্যনাটক বাংলায় থুব বেশী চচচচা হয়নি। আমি সেটাকেই অন্য আঙ্গিকে প্রকাশ করতে চেয়েছি। শুধু নিজে লিথেছি তাই নয়, আমি আমার পরবর্তী প্রজন্মের ছেলেমেয়েদের দিয়েও লিথিয়েছি। যেমন পরবর্তীকালে মন্দাক্রান্তা সেন বেশ কয়েকটি কাব্যনাটক লিথেছ্েেন। তাকে দিয়ে আমিই লিথিয়েছিলাম, মানে একদম জোর করে ধরে লিথিয়েছিলাম বলা যেতে পারে।সম্প্রতি আমি আবার বেশকিছু তরুনদের দিয়ে লিখিয়েছি। কাব্যনাট্য প্রযোজনার কথা ভেবেছ্,ি, কাব্যনাটক নিয়ে সেমিনার করেছ্ছি, কাব্যনাটক নিয়ে সংখ্যা সম্পাদনা করেছি। So

\section{I got myself in various aspects of verse drama/poetic dramal}

শ্রীতন্বী চঞবর্তীঃ আপনার জীবনের গঠনমূলক পর্যায়ে উত্তরবঙ্গের অবদান অগরিসীম। আপনার বেশ কিছু কাজে উত্তরবঙ্গ শুধু একটি স্থান নয়, বরং একটি প্রতীয়মান চরিত্র হিসেবে ধরা দেয়।

প্রবাল কুমার বসুঃ উত্তরবঙ্গে আমার যথন পড়ার সুযোগ এলো, আমাকে অনেকেই বলেছিল উত্তরবঙ্গে যেওনা, ওখানে পড়াশুনা হয়না। ওথানে গেলে ছেলেরা নষ্ট হয়ে যায়। কিন্তু আমি জোর করে গিয়েছিলাম। এবং তারপর সেখান থেকে পাশ করে বেরিয়ে, এবং এখনো যথন পিছ্ন ফিরে দেথি, ওই সময়টা আমার কাছে একটা স্বগ্নের মতো। তারপরেও প্রায় প্রতি বছুরই একসময় যেতাম, এখনো, প্রতি বছর নাহলেও এক-দু বছর অন্তর যাই। উত্তরবঙ্গ আমাকে প্রকৃতির কাছাকাছ্ এনে দিয়েছিলো। আমার জীবনে প্রকৃতিকে দেথার দৃষ্টিভঙ্গি খুলে দিয়েছিলো উত্তরবঙ্গ। আমি যেভাবে উত্তরবঙ্গ ঘুরেছ্, আমার সঙ্গে অনেকবার থেকেছেন শক্তি চটেটোপাধ্যায়, ধরে ধরে আমাকে গাছ চিনিয়েছেন, জঙ্গল চিনিয়েছেন, এটা থেকে বলতে গারি, উত্তরবঙ্গ আমার জীবনে পরম প্রাপ্তি। দু-একটা প্রেমও যে উত্তরবঙ্গে হয়নি তা নয়, সেটাও প্রাপ্তি।

শ্রীতন্বী চঞ্র্তীঃ অনুবাদ এবং বহুভাযিক উপাদান আমাদের সাহিত্যকে কিভাবে সমৃদ্ধ করেছে? অনুবাদ সাহিত্য নিয়ে আभনার ব্যক্তিগত মতামত কি?

প্রবাল কুমার বসুঃ অনুবাদ সম্বন্ধে যেটা বলা হয়, এবং যেটা আমিও বিশ্বাস করি, বিশেষত কবিতা অনুবাদের ব্যাগারে, যে এক ঝাঁকা আম, তুমি বম্বে থেকে

Note: This is an interview conducted in Bengali and there is no citation or works cited required. It is a live interview taken by Sreetanwi Chakraborty, Chief-Editor of Litinfinite Journal. 


\section{Litinfinite Journal}

ISSN: 2582-0400 [Online]

CODEN: LITIBR

Vol-1, Issue-1 ( $2^{\text {nd }}$ July, 2019)

Page No: $78-88$

DOI: 10.47365/litinfinite.1.1.2019.78-88

\section{Section: Interview}

কলকাতা পাঠালে, ঝাঁকাটা এসে পৌঁছছল, আমগুলো এলো না। অনুবাদকেরা, যারা অনুবাদ করেন, তারা অনেক সময় translation কে transcreation 3 বলতে চেয়েছেন। আমাদের দেশের পরিপ্রেক্ষিতে translation এর কাজ থুব একটা হয় না। আমাদের history of civilization হছে history of translation 1 আমরা যথন আস্তে আস্তে সভ্য হতে শুরু করলাম, তথন আমরা ছবি আঁকতাম, ছবি এঁকে communicate করতাম। মানে আমি বাঘ বলতে চাইলাম, বাঘ আঁকলাম, বাঘ বোঝা গেলো, এটাও তো একধরনের translation। সাহিত্যের translation জরুরী। নাহলে বিদেশী সাহিত্য কি লেথা আমরা পড়তে পারতাম না। স্প্যানিশ সাহিত্য পড়তে পারতাম না, লোরকা পড়তে পারতাম না। ব্রিটিশরা আমাদের এখানে থাকার দরুন, আমরা ইংরেজিটা একটু আধটু জানি। কিন্ত স্প্যানিশ সাহিত্য যে থুব সমৃদ্ধ তা আমরা কজন জানি? আমরা Marquez পড়তে পারতাম না, অনুবাদ নাহলে। ফলে অনুবাদ করাটা ভীযণ জরুরী। কিন্ত বিদেশে যেভাবে অনুবাদ হয়, অর্থাৎ অনুবাদকের একটা ফুলটাইম জব, এবং সে যে লেথকের অনুবাদ করছে, তার সঙ্গে আলোচনা করে, সময় কাটায়, কাটিয়ে সে একটা অনুবাদের কাজ তৈরি করে। আমাদের দেশে সেটা হয় না। আমি কি লিথলাম, সেটা যথন একজন অনুবাদ করতে যাছে, তথন সে তার মতো অনুবাদ করে। ফলে আমাদের সাহিত্য কখনই কোন বিদেশী মাপকাঠিতে দৌঁছয়ই না। ফলে আমাদের সাহিত্য কথনই বিশ্বের কোন বড় পুরষ্কার পাবার আায়গায়, পৌঁছ্য়ই না, এবং পৌঁছবেও না আগাতত।আমাদের সামাজিক এবং অর্থনৈতিক পরিকাঠামো সেই অবকাশটা দেয় না। ফলে আমাদের লেথা যে অনুবাদ হচ্ছেনা, তা নয়, অনুবাদ হচ্ছে, হয়ও, কিন্ত সেই লেথা এমন কোন পর্যায়ে পৌঁছয় না, যে সেটা বিশ্বের লেথার সাথে তুলনীয় হতে পারে। আমরা রবীন্দ্রনাথের প্রসঙ্গ আনি সব বিষয়েই।রবীন্দ্রনাথের ওই অনুবাদ যদি না W.B. Yeats দেথে না দিতেন, এবং এডিট না করে দিতেন, ওটা গাঠযোগ্য হতো না। রবীন্দ্রনাথের সৌভাগ্য ছিল যে উনি W.B. Yeats এবং Ezra Pound এর সান্নিধ্য পেয়েছিলেন। এথন সেরকম কোন লেথক ভারতবর্মে তো নেই, বাংলাতেও নেই, যে এই বিদেশী সাহচর্য পেতে পারে। ফলে থুব বড় কোনো জায়গায় অনুবাদ পৌঁছনোর কোনো অবকাশ নেই। সাহিত্যরসে উত্তীর্ণ হওয়ার মতো এই মুহূত্তেই বিশেষ কিছ্ডু সম্ভাবনা নেই বলেই আমার মনে হয়, যদিও অনুবাদকেরা ভিন্নমত নিশ্চয়ই পোযণ করবেন।

শীরীন্বী চঞ্রবর্তীঃ আগনার কিছু কিছু কবিতায় এবং কাব্যনাটকে আগুন, মৃত্যু, পৃথিবী এবং জল বিশেষ বিশেষ কিছু অনুষঙ্গ হিসেবে প্রকাশ পায়। এই প্রাকৃতিক উপাদানগুলো কি কোনভাবে অস্তিত্বের সঙ্কট নিয়ে ভাবতে শেখায়?

প্রবাল কুমার বসুঃ আমার মনে হয় না যে আমি থুব pessimistic ভাবনা নিয়ে লিখি। আমার লেখা সার্বিকভাবে pessimistic নয় কথনো। আর যে প্রকৃতির অনুযঙ্গ গুলোর কথা বলা হছে, সেগুলো দীর্ঘদিন উত্তরবঙ্গে প্রকৃতির কাছাকাছ্ থাকার প্রাগ্তি। সেটাই নানাভাবে ঘুরেফিরে আমার কবিতায় প্রকাশ পেয়েছে। আর একজন কবির তো exactly a journey to reach to oneself that is ultimately poetry / One never reaches, but the journey continues। এই পথ চলার সময় নানারকম অনুষঙ্গ আসে চারপাশ থেকে। আমার লেথায় সার্বিকভাবে দুটো বিষয়ের অনুষঙ্গ আছে। একটা প্রকৃতি, উত্তরবঙ্গে যার বিস্তার, আর 
Litinfinite Journal

ISSN: 2582-0400 [Online]

CODEN: LITIBR

Vol-1, Issue-1 (2 $\left.2^{\text {nd }} J u l y, 2019\right)$

Page No: 78-88

DOI: 10.47365/litinfinite.1.1.2019.78-88

Section: Interview

একটা হছে সম্পর্ক। এই সম্পর্কটাও আসলে নিজের সঙ্গে নিডের সম্পর্ক। হয়তো কাউকে কেন্দ্র করে, হয়তো বিভিন্নজনকে কেন্দ্র করে, কিন্ত সর্বশেশে নিডোর সঙ্গে নিডেরই সম্পর্ক।

শ্রীতন্বী চঞ্র্তীঃ যাপনচিত্রর সূচনা এবং কার্যকলাপ সম্বন্ধে কিছু বলুন।

প্রবাল কুমার বসুঃ যাপনচিত্র একটা পত্রিকা। মনে হয়েছিলো, যে তরুনদের জন্যে আক্করিক অর্থে, যে সময় শুরু করি সেই সময় সামগ্রিকভাবে তরুণদের লেখালিথি, তরুণদের ভাবনাকে প্রকাশ করার কাগজ কৃত্তিবাস ছাড়া আর কিছুই ছিল না। ক্রিয়েটিভ পত্রিকা যাকে বলি, সেরকম কিজুই ছিল না সেই সময়। সেই ভাবনা থেকেই যাপনচিত্র শুরু করা হয়।যাপনচিত্র মূলত কবিতার কাগজ হলেও, শিল্প-সংস্কৃতির অন্যান্য সমস্ত বিষয়েও কাজ করেছে। যাপনচির্র বৈশিষ্ট্য হছে ক্রোড়পত্র। সমাজভাবনা, নাটক, শিল্পীদের আঁকা ছবি, याত্রা, থিয়েটার, ফিল্ম, দীর্ষ কবিতা- সমস্তু রকম বিষয় নিয়েই কাজ করেছে যাগনচিত্র। এবং এতদিনে মানুমের মনে, নিজস্ব একটি স্থান নিজগুনে তৈরি করতে পেরেছে বলে মনে হয়।

শ্রীতন্বী চঞ্র্তীঃ নতুন প্রজন্মের কবিদের প্রতি কোন পরামর্শ দেবেন?

প্রবাল কুমার বসুঃ নতুন কবিরা নিজগুনে অত্যন্ত সমৃদ্ধ। শুধু একটি উপদেশ - তারা ছুন্দটা একটু ঠিকঠাক শিথুক। শুধু পুরষ্কার আর লেথা ছাপানোর পিজ্নে না দৌড়ে, নিজেদের লেখাটা যাতে ঠিকসত লিথতে গারে সেই চেষ্টা করুক।

कবिতा

\section{প্রেম भুনরায়}

প্রবাল কুমার বসू

Note: This is an interview conducted in Bengali and there is no citation or works cited required. It is a live interview taken by Sreetanwi Chakraborty, Chief-Editor of Litinfinite Journal. 
Litinfinite Journal

ISSN: 2582-0400 [Online]

CODEN: LITIBR

Vol-1, Issue-1 ( $2^{\text {nd }}$ July, 2019)

Page No: $78-88$

DOI: 10.47365/litinfinite.1.1.2019.78-88

Section: Interview

সন্দেহের উর্দের আমি নই

यদিও অনুষ্চারিত

ভেবেছ্ বাতিল করে দেবে

ভেবেছ্ সেটাই সঙঁগঁ?

তাই এড়িয়ে গিয়েছ্ অকারণে

এড়িয়ে গিয়েছ বারবার

উপেক্ষা করেছ্ গিয়ে যত

বেড়ে গেছে আহ্মবিশ্বাস

শেষমেশ করেছ্ উপাসনা

জমিয়ে রেথে সায়াফ্নের আলো

এসব কথা তোমার কাছেই শোনা

শুধু বলতে গিয়েই অযথা আটকালো

\section{লেখার কথা}

প্রবাল কুমার বসু

অল্পই লিথেছ্

যথনই লিথেছি সেই লেথা

বজ্রপাতে গুড়ে গেছে

ছাই আর ধুলোমাখা থাতা

সেই ছাই ধুলো ঘেঁটে ঘেঁটে

কি লিথেছ্ যথনই কেউ দেখতে চেয়েছ্ছে

দেথেছে অক্ষর শুষ্নু

তাও তার মাথাগুলো কাটা

অল্পই লিথেছ্ছি

লিথতে লিথতে শুধু

ভেঙেছি স্বুব্ধতা

Note: This is an interview conducted in Bengali and there is no citation or works cited required. It is a live interview taken by Sreetanwi Chakraborty, Chief-Editor of Litinfinite Journal.

\section{Suggested list of reading:}

Basu, P. K. Ontoraler Golpo Kotha. KOLKATA, None: Atmajaa, 2019. Print. 
Litinfinite Journal

ISSN: 2582-0400 [Online]

CODEN: LITIBR

Vol-1, Issue-1 ( $2^{\text {nd }}$ July, 2019)

Page No: $78-88$

DOI: 10.47365/litinfinite.1.1.2019.78-88

Section: Interview

Basu, Prabālakumāra. Signposts: Bengali Poetry Since Independence. books catalog, 2002.

Print.

Basu, Prabālakumāra. Yapanchitra, a Profile of Life: An Issue on International Poetry. N.p., 2006. Print.

"Prabal Kumar Basu - Times of India." The Times of India. N.p., n.d. Web. 17 Aug. 2018.

"Prabal Kumar Basu." Wikitia. N.p., n.d. Web. 2 May 2018.

<https://wikitia.com/wiki/Prabal_Kumar_Basu>.

Basu, Prabal Kumar. Ei Je Ami Cholechi (Bengali Edition). Ananda Publishers, 2015.

Note: This is an interview conducted in Bengali and there is no citation or works cited required. It is a live interview taken by Sreetanwi Chakraborty, Chief-Editor of Litinfinite Journal. 